\title{
Efeitos antitussígenos e expectorantes de dois fitoterápicos comercializados no mercado brasileiro*
}

\author{
Antitussive and expectorant effects of two phytotherapics in the brazilian market
}

Fernanda Bastos de Mello' \& João Roberto Braga de Mello²

\begin{abstract}
RESUMO
Os efeitos antitussígenos - expectorantes de dois fitoterápicos existentes no mercado brasileiro, foram avaliados utilizando-se três modelos biológicos diferentes. Cada formulação fitoterápica, com composição diferente, apresentava o mesmo número de lote e data de fabricação. Os nomes comerciais dos fitoterápicos avaliados foram: Extrato Expectorante Salva e Xarope Expectorante e Sedativo da Tosse Fitomed. Uma espécie animal específica foi utilizada em cada modelo avaliado (n=10 animais/grupo). Foram utilizados ratos Wistar no modelo da secreção das vias aéreas, cobaios no modelo de tosse induzido por ácido cítrico e codornas japonesas na determinação da velocidade de transporte mucociliar. Os animais (um grupo/formulação) foram divididos nos dois grupos de fitoterápicos e tratados por via oral, com o equivalente a dez vezes a dose terapêutica recomendada $\left(9 \mathrm{~mL} \cdot \mathrm{kg}^{-1}\right)$. Um grupo controle negativo de cada espécie foi tratado com solução fisiológica $\left(10 \mathrm{~mL} \cdot \mathrm{kg}^{-1}\right)$. Um grupo controle positivo composto por cobaios utilizados no modelo de tosse induzida pelo ácido cítrico foi tratado com morfina $\left(1 \mathrm{mg} \cdot \mathrm{kg}^{-1}\right.$ ) por via subcutânea. No modelo onde foram utilizados ratos (secreção das vias aéreas) e codornas (determinação de velocidade de transporte mucociliar) o grupo controle positivo recebeu erdosteína por via oral $\left(600 \mathrm{mg} \mathrm{kg}^{-1}\right)$. Os resultados mostraram que as duas formulações fitoterápicas foram eficazes em inibir o reflexo da tosse induzido pelo ácido cítrico em cobaios. Nos modelos de secreção das vias aéreas em ratos e determinação da velocidade de transporte mucociliar em codornas nenhuma das duas formulações apresentou eficácia significativa.
\end{abstract}

Descritores: Mikania glomerata, Salvia officinalis, Eucalyptus globulus, Myrospermum erytroxilon, Nasturtium officinale, Copaifera langsdorffi.

\section{ABSTRACT}

The antitussive-expectorant effects of two phytotherapic formulations of Brazilian market were evaluated using three different biological models. Each phytotherapic formulation, with different composition, had the same batch number and fabrication date. The trade names of phytotherapics were: Extrato Expectorante Salva and Xarope Expectorante e Sedativo da Tosse Fitomed. A specific animal specie was used in each evaluated model ( $\mathrm{n}=10$ animals/group). Wistar rats in the airway secretion model, guinea pigs in the citric acid-induced cough model and japanese quails in the mucociliary trans-port rate determination were used. The animals (one group/formulation) were divided in the two phytotherapic groups, and treated orally with the equivalent to ten times the therapeutic recommended dose $\left(9 \mathrm{~mL} . \mathrm{kg}^{-1}\right)$. A negative control group each specie was treated orally with saline $\left(10 \mathrm{~mL} \cdot \mathrm{kg}^{-1}\right)$. Positive guinea-pigs citric acid-induced cough model was treated with morphine ( $\left.1 \mathrm{mg}^{\mathrm{kg}}{ }^{-1}\right)$ subcutaneously. In the rat airway secretion model and japanese quails mucociliary transport rate, a positive control received orally erdosteine $\left(600 \mathrm{mg} \cdot \mathrm{kg}^{-1}\right)$. The results showed that the six phytotherapic formulations were able to inhibit the guinea pig cough reflex induced by citric acid. The rat airway secretion and the mucociliary transport rate were not significantly affected by the two phytotherapic formulations.

Key words: Mikania glomerata, Salvia officinalis, Eucalyptus globulus, Myrospermum erytroxilon, Nasturtium officinale, Copaifera langsdorffi. 


\section{INTRODUÇÃO}

O aparelho respiratório possui alguns mecanismos que desempenham uma função de defesa e limpeza da árvore respiratória, tais como o reflexo da tosse e o sistema mucociliar $[1,7,10,18]$.

A tosse e o reflexo de broncoconstrição, manifestações comuns de doença respiratória [16], podem ser gerados por estimulação mecânica das vias aéreas, inalantes irritantes ou liberação local de mediadores agindo nos receptores de adaptação rápida e nas terminações nervosas das fibras $\mathrm{C}$ presentes na laringe e mucosa traqueobrônquica [22].

$\mathrm{O}$ transporte mucociliar, outro mecanismo de defesa pulmonar, serve para remover partículas inaladas da mucosa traqueobrônquica [21]. O sistema mucociliar é responsável pela movimentação de fluidos (muco), os quais são produzidos pelas células caliciformes e pelas glândulas brônquicas [8,13,14].

Teoricamente o mecanismo pelo qual há a interação do transporte mucociliar, está diretamente relacionado com as alterações na quantidade e qualidade das secreções epiteliais (fluido periciliar e muco) e alterações na integridade anatômica e funcional dos cílios [20].

As doenças relacionadas com o aparelho respiratório são de alta incidência entre os animais de estimação, onde o emprego adequado de substâncias na terapia instituída é o ponto chave para o êxito do tratamento e para o bem estar do paciente. Para o tratamento das doenças respiratórias, utilizam-se diversos fármacos, principalmente antitussígenos, expectorantes, antiasmáticos e tensoativos pulmonares, nem sempre com eficácia comprovada [11].

O objetivo do trabalho foi avaliar duas apresentações fitoterápicas existentes no mercado brasileiro frente a esses três modelos biológicos distintos.

\section{MATERIAIS E MÉTODOS}

\section{Animais}

Foram utilizados 40 ratos albinos Wistar machos com idade em torno de 100 dias (com massa corporal de 326,6 $44,9 \mathrm{~g}$ ), provenientes do Centro de Criação e Experimentação de Animais de Laboratório da UFRGS (CREAL), 40 cobaios albinos da linhagem Short Hair machos com idade em torno de 100 dias (com massa corporal de 693,4 $\pm 14,2 \mathrm{~g}$ ), provenientes do Laboratório Animal de Apoio Regional (LARA/RS) e 40 codornas japonesas (Coturnix coturnix japonica) machos com idade em torno de 40 dias (com massa corporal de 147,0 $\pm 2,5 \mathrm{~g}$ ), provenientes de estabelecimento comercial ${ }^{3}$. Os animais foram mantidos no Biotério Setorial do Departamento de Farmacologia do Instituto de Ciências Básicas da Saúde (ICBS) com condições constantes de umidade, temperatura $\left(21^{\circ} \mathrm{C} \pm 2\right)$ e ciclo de luz claro/escuro de 12 horas (claro das $9 \mathrm{~h}$ às 21h). Foram alimentados com ração comercial e água ad libitum durante todo o período experimental. Os animais foram aclimatados às condições do Biotério Setorial por um período mínimo de 15 dias.

\section{Apresentações farmacêuticas fitoterápicas empregadas}

Foram utilizadas as apresentações farmacêuticas que contêm em sua formulação uma única planta ou extrato de plantas em associação e indicados como antitussígeno-expectorantes, coadjuvantes no tratamento das afecções do aparelho respiratório.

Os fitoterápicos utilizados nos experimentos foram adquiridos de estabelecimentos farmacêuticos contendo cada um especificamente o mesmo número de lote e data de fabricação e estando dentro do prazo de validade indicado pelo fabricante. Foram utilizadas as seguintes apresentações farmacêuticas:

Extrato Expectorante Salva ${ }^{1}$, lote: 0002, produzido em junho de 2001 pelo Laboratório Industrial Vida e Saúde Ltda. (Chapecó/SC), com validade até junho de 2004. Registrado no Ministério da Saúde sob o n ${ }^{\circ}$ 1.02.838.8.

Xarope Expectorante e Sedativo da Tosse Fitomed ${ }^{2}$, lote: 080271, produzido em julho de 2001 pelo Herbarium Laboratório Botânico Ltda. (Colombo/PR), com validade até julho de 2004. Registrado no Ministério da Saúde sob n ${ }^{\circ} 1.1860 .0268$.

\section{Composição quali-quantitativa dos fitoterápicos}

Extrato Expectorante Salva ${ }^{1}$ - cada 100 mL contém:

Extrato fluido de guaco (Mikania glomerata) ............... 4,000 mL Extrato fluido de salva (Salvia officinalis) ..................... 2,000 mL Extrato fluido de eucalipto (Eucalyptus globulus) ........ 2,000 mL Extrato fluido de gengibre (Zingiber officinalis) ........... 0,200 mL Extrato fluido de própolis .............................................. 1,000 mL Veículo q.s.p. .............................................................. $100 \mathrm{~mL}$

Xarope Expectorante e Sedativo da Tosse Fitomed ${ }^{2}$ - cada $\mathrm{mL}$ contém:

Tintura de grindelia (Grindelia sp.) …………………..... $0,01 \mathrm{~mL}$ Tintura de guaco (Mikania glomerata) ............................ $0,10 \mathrm{~mL}$ Tintura de bálsamo de tolú (Myrospermum erytroxylon) .... 0,01 mL Tintura de própolis …………………………………... 0,005 mL Extrato fluido de agrião (Nasturtium officinale) ................ 0,05 mL 
Óleo de copaíba (Copaifera langsdorffi) ……………... 0,001 mL

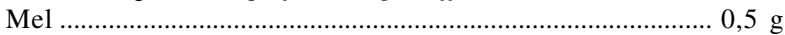
Excipiente q.s.p. …………………………………... 1,000 mL

\section{Fármacos utilizados nos protocolos experimentais}

Nos protocolos experimentais onde ratos e codornas foram usados como modelo biológico, a erdosteína foi utilizada como fármaco com atividade mucolítica comprovada [9], possibilitando a constituição de grupos controle-positivos. Foi utilizada a apresentação farmacêutica Flusten ${ }^{4}$ cápsulas $300 \mathrm{mg}$ de erdosteína, lote: 002/02, produzido em julho de 2002 pelo Laboratório Eurofarma Ltda. (São Paulo/SP), com validade até julho de 2004. Registrado no Ministério da Saúde sob n ${ }^{\circ}$ 1.0043.0611.

Para constituir o grupo controle positivo no protocolo experimental cujo modelo biológico foi o cobaio, o cloridrato de morfina ${ }^{5}$ foi usado como fármaco com atividade antitussígena comprovada [6].

\section{Avaliação da secreção das vias aéreas em ratos}

O método utilizado para avaliação quantitativa da secreção foi o método de Kohda modificado [9].

Foram utilizados dez ratos albinos Wistar machos por grupo. Os animais foram pesados individualmente e tratados por via oral (gavagem), com o auxílio de uma sonda flexível, com as apresentações farmacêuticas selecionadas. A dose utilizada foi correspondente a dez vezes a dose terapêutica recomendada pelo fabricante cuja tolerância ao volume e efeitos terapêuticos foram avaliados em ensaio piloto.

Os grupos controles negativo e positivo foram constituídos por igual número de animais ( $\mathrm{n}=10$ /grupo) e foram tratados com solução fisiológica $\left(10 \mathrm{~mL} \cdot \mathrm{kg}^{-1}\right) \mathrm{e}$ erdosteína (600 $\left.\mathrm{mg} \cdot \mathrm{kg}^{-1}\right)$, respectivamente, da mesma maneira como citado anteriormente.

Uma hora após a administração oral das apresentações farmacêuticas avaliadas no experimento, os animais foram anestesiados com uma associação de cloridrato de tiletamina e cloridrato de zolazepam ${ }^{6}$ por via intramuscular. Foi realizada a punção do vaso sanguíneo e a administração de fenolsulfoftaleína ${ }^{7}\left(6 \mathrm{mg} \cdot \mathrm{kg}^{-1}\right)$.

Trinta minutos após a administração do corante, os animais foram eutanasiados, e foi realizado lavado broncoalveolar com $5 \mathrm{~mL}$ de $\mathrm{NaHCO}_{3} 5 \%$.

$\mathrm{O}$ lavado bronco alveolar foi centrifugado com velocidade de $3000 \mathrm{rpm}$ durante 30 minutos. $\mathrm{O} \mathrm{pH}$ do sobrenadante de cada lavado bronco alveolar foi ajustado em 8.0, após a centrifugação, através da adi- ção de uma solução de HCL 1M. A absorbância de cada lavado bronco alveolar foi determinada em espectrofotômetro U/V visível, no comprimento de onda de $558 \mathrm{~nm}$.

\section{Avaliação do reflexo da tosse induzido pelo ácido cítrico em cobaios}

Para a avaliação do reflexo da tosse foi utilizado método de Miyata, com modificações [9].

Os animais foram divididos em grupos experimentais distintos, sendo cada um deles constituído por dez animais, e identificados de forma inequívoca. Os animais foram colocados individualmente, de forma seqüencial, em uma câmara de inalação acrílica transparente de exposição de corpo inteiro $(20 \times 20 \times 20 \mathrm{~cm})$ e expostos a aerossóis de ácido cítrico 5\%, durante 5 minutos consecutivos.

Durante o período de exposição o número de episódios de tosse apresentado por cada animal foi contado e registrado

Três horas após essa exposição, os animais foram tratados, por via oral, com as apresentações farmacêuticas, com dose equivalente a dez vezes a dose terapêutica recomendada pelo laboratório fabricante, por meio de uma sonda oral rígida.

Uma hora após o tratamento por via oral foi realizado mo mesmo procedimento de exposição e registro de tosse. Os grupos controles também foram expostos aos aerossóis de ácido cítrico. O controle negativo foi tratado com solução fisiológica $\left(10 \mathrm{~mL} \cdot \mathrm{kg}^{-1}\right)$ e no grupo controle positivo foi administrado cloridrato de morfina ${ }^{5}\left(1 \mathrm{mg} \cdot \mathrm{kg}^{-1}\right)$ por via subcutânea.

\section{Determinação da velocidade de transporte mucociliar na traquéia de codornas}

As aves foram dispostas aleatoriamente em grupos experimentais distintos, constituídos cada um por 10 animais. Os animais foram pesados e tratados, por via oral, com o auxílio de uma sonda oro-gástrica flexível, com as apresentações farmacêuticas utilizadas. Os animais que constituíram os grupos controles, negativo e positivo, foram tratados de maneira idêntica aos demais. No grupo controle negativo foi administrado solução fisiológica $\left(10 \mathrm{~mL} \cdot \mathrm{kg}^{-1}\right)$ e no grupo controle positivo foi administrado erdosteína (600mg. $\mathrm{kg}^{-1}$ ).

Após uma hora da administração oral das apresentações farmacêuticas avaliadas no experimento, os animais foram anestesiados com uma associação de 
cloridrato de tiletamina e cloridrato de zolazepam ${ }^{6}$ na dose de $50 \mathrm{mg} . \mathrm{kg}^{-1}$ por via intramuscular.

Após a aquisição de plano anestésico cirúrgico, a traquéia foi localizada e exposta cuidadosamente. A região adjacente à traquéia foi recoberta com gaze umedecida em solução fisiológica aquecida $\left(37^{\circ} \mathrm{C}\right)$. A traquéia foi incisada longitudinalmente $(5 \mathrm{~mm})$. Após a abertura desse órgão, os animais foram colocados individualmente sob um microscópio esterioscópico com aumento de 0,7 a quatro vezes, para avaliação da velocidade do transporte mucociliar. Uma pequena e fina partícula de carvão vegetal ativado foi introduzida na camada mucosa da traquéia (partícula marcadora), na região proximal à cavidade oral, e o tempo decorrente da sua movimentação nos cinco milímetros estabelecidos no tamanho da incisão, foi cronometrado e registrado. $\mathrm{O}$ movimento mucociliar foi avaliado de 10 em 10 minutos durante 120 minutos consecutivos.

Durante o intervalo de tempo entre cada aferição da velocidade do transporte mucociliar, a região cervical dos animais foi coberta com compressas de gaze umedecidas com solução fisiológica aquecida a $37^{\circ} \mathrm{C}$, e os animais aquecidos com uma lâmpada auxiliar.

\section{Análise estatística}

A metodologia utilizada para análise estatística incluiu os testes: análise de variância de uma via (ANOVA), análise de variância de medidas repetidas (ANOVA de MR) e Teste de Bonferroni [2,23].

As variáveis quantitativas que apresentaram distribuição normal referentes a secreção das vias aéreas em ratos e a indução do reflexo da tosse em cobaios foram comparadas através da análise de variância de uma via (ANOVA).

As variáveis quantitativas que apresentaram distribuição normal referentes a velocidade de transporte mucociliar em codornas foram comparadas através da análise de variância de medidas repetidas (ANOVA MR).

Todas as variáveis respeitaram os valores estatisticamente significativos, com uma confiança de $95 \%$ ( $\mathrm{p}=$ $0,05)$. Foram citados os níveis de significância $(\alpha)$ alcançados em cada teste estatístico realizado.
Os programas utilizados para efetuar a análise estatística foram o SPSS for Windows 8.0 e o EXCEL 4.0 [12].

Com relação as variáveis quantitativas, sempre que se fez necessário a comparação entre médias, foi utilizado o Teste de Bonferroni.

\section{RESULTADOS}

\section{Avaliação da secreção das vias aéreas em ratos}

A Figura 1 mostra a concentração de corante $(\mathrm{mg} / \mathrm{mL})$ obtida no lavado broncoalveolar de ratos pertencentes aos grupos tratados com o Extrato Expectorante Salva ${ }^{1}$ e Xarope Expectorante e Sedativo da Tosse Fitomed ${ }^{2}$, grupo controle negativo tratado com o veículo (solução fisiológica) e grupo controle positivo tratado com erdosteína (Flusten ${ }^{4}$ ) na dose de $600 \mathrm{mg} \cdot \mathrm{kg}^{-1}$. Com base nos resultados, verifica-se que houve diferença estatisticamente significativa entre os grupos (ANOVA: $\mathrm{p}=0,011)$. Com a realização do Teste de Bonferroni, conclui-se que o grupo controle positivo é estatisticamente diferente do grupo controle negativo e do grupo tratado com o Extrato Expectorante e Sedativo da Tosse Fitomed ${ }^{2}$.

\section{Avaliação do reflexo da tosse induzido pelo ácido cítrico em cobaios}

A Tabela 1 apresenta o número de eventos de tosse apresentado pelos animais expostos a aerossóis de solução aquosa de ácido cítrico $5 \%$ após as duas exposições seqüenciais, pertencentes aos grupos tratados

$\mathbf{Q}$ Xarope Expectorante e Sedativo da Tosse Fitomed 回Extrato Expectorante Salva

Controle positivo

$\checkmark$ Controle negativo

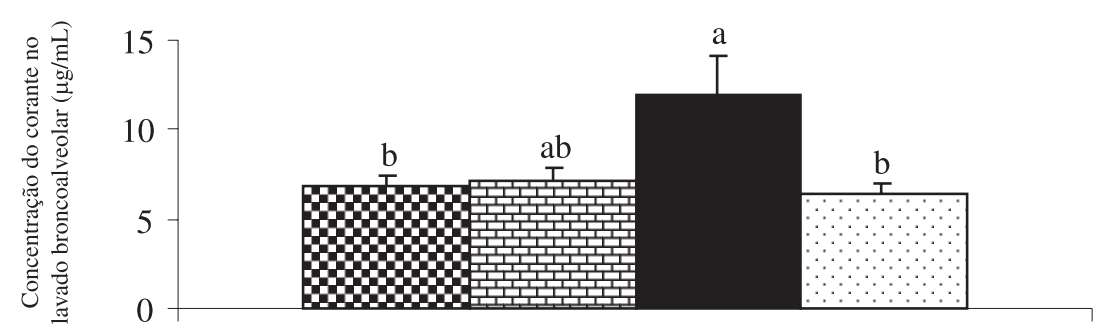

Figura 1. Concentração de fenolsulfoftaleína $(\mu \mathrm{g} / \mathrm{mL})$ obtida no lavado broncoalveolar de ratos pertencentes ao grupo tratado com Extrato Expectorante Salva, Xarope Expectorante e Sedativo da Tosse Fitomed, grupo controle negativo tratado com o veículo (solução fisiológica) e grupo controle positivo tratado com Flusten (erdosteína). Os valores representam a média dos grupos e as barras verticais representam o epm ( $\mathrm{n}=10$ animais/grupo). Letras diferentes indicam diferença estatisticamente significativa $(\mathrm{p}<0,05)$. 
Tabela 1. Número absoluto e porcentual de modificação de eventos de tosse, apresentado pelos animais expostos a aerossóis de solução aquosa de ácido cítrico 5\% após as duas exposições seqüenciais (3 horas antes do tratamento e 1 hora após). São apresentados os valores médios \pm epm para cada grupo experimental ( $\mathrm{n}=10$ animais/grupo).

\begin{tabular}{lccc}
\hline \multirow{2}{*}{ Grupo } & \multicolumn{2}{c}{ Número absoluto de eventos de tosse } & \multirow{2}{*}{ Porcentual (\%) } \\
\cline { 2 - 3 } & Primeira exposição & Segunda exposição & \\
\hline Extrato Expectorante Salva & $14,5 \pm 2,38$ & $6,1 \pm 1,42$ & $-66,2 \pm 9,09^{*}$ \\
$\begin{array}{l}\text { Xarope Expectorante } \mathrm{N} \\
\text { Sedativo da Tosse Fitomed }\end{array}$ & $10,7 \pm 1.54$ & $6,9 \pm 1,57$ & $-34,3 \pm 12,27^{*}$ \\
$\begin{array}{l}\text { Controle negativo } \\
\text { Controle positivo }\end{array}$ & $11,3 \pm 1,13$ & $15,4 \pm 2,48$ & $+20,6 \pm 8,83$ \\
$\begin{array}{l}\text { "Diferença estatisticamente significativa (p<0,05).No valor porcentual, o sinal positivo indica aumento no número } \\
\text { de eventos apresentados e o sinal negativo indica redução no número de eventos apresentados, tendo-se a primeira } \\
\text { exposição como referência. }\end{array}$ &
\end{tabular}

com Extrato Expectorante Salva ${ }^{1}$, Xarope Expectorante e Sedativo da Tosse Fitomed ${ }^{2}$, grupo controle negativo tratado com o veículo (solução fisiológica) e grupo controle positivo no qual foi administrado cloridrato de morfina ${ }^{4}\left(1 \mathrm{mg} \cdot \mathrm{kg}^{-1}\right)$ por via subcutânea.

Com relação à análise estatística do percentual de redução entre os três grupos de tratamento, verificase que houve diferença estatisticamente significativa entre eles (ANOVA: $\mathrm{p}<0,001$ ). Com a realização do Teste de Bonferroni, conclui-se que os grupos controle positivo, Extrato Expectorante Salva ${ }^{1}$, Xarope Expectorante e Sedativo da Tosse Fitomed ${ }^{2}$ são estatisticamente diferentes do grupo controle negativo.

\section{Determinação da velocidade de transporte mucociliar na traquéia de codornas}

A Figura 2 mostra a velocidade do transporte mucociliar aferida através da adição de uma partícula marcadora na traquéia das codornas pertencentes aos grupos tratados com os com Extrato Expectorante Salva ${ }^{1}$, Xarope Expectorante e Sedativo da Tosse Fitomed ${ }^{2}$, grupo controle negativo tratado com o veículo (solução fisiológica) e grupo controle positivo tratado com erdosteína (Flusten $^{4}$ ) na dose de $600 \mathrm{mg} \cdot \mathrm{kg}^{-1}$. Com base nos resultados, verifica-se que houve diferença estatisticamente significativa entre os grupos em alguns tempos medidos (ANOVA de MR: $\mathrm{p}=0,025$ ). Com a realização do Teste de Bonferroni, conclui-se que o grupo controle positivo é estatisticamente diferente do grupo controle negativo nas medidas realizadas aos 80, 90, 100,110 e 120 minutos.

Embora tenha ocorrido aumento na velocidade de transporte mucociliar no grupo tratado com Extrato Expectorante Salva ${ }^{1}$, o aumento não foi estatisticamente significativo.

\section{DISCUSSÃO}

Há muitos mecanismos relacionados com a expectoração. Expectorantes atuam por redução da viscosidade do muco, promover a secreção dos fluidos do trato respiratório e surfactantes e normalização do muco traqueal [9].

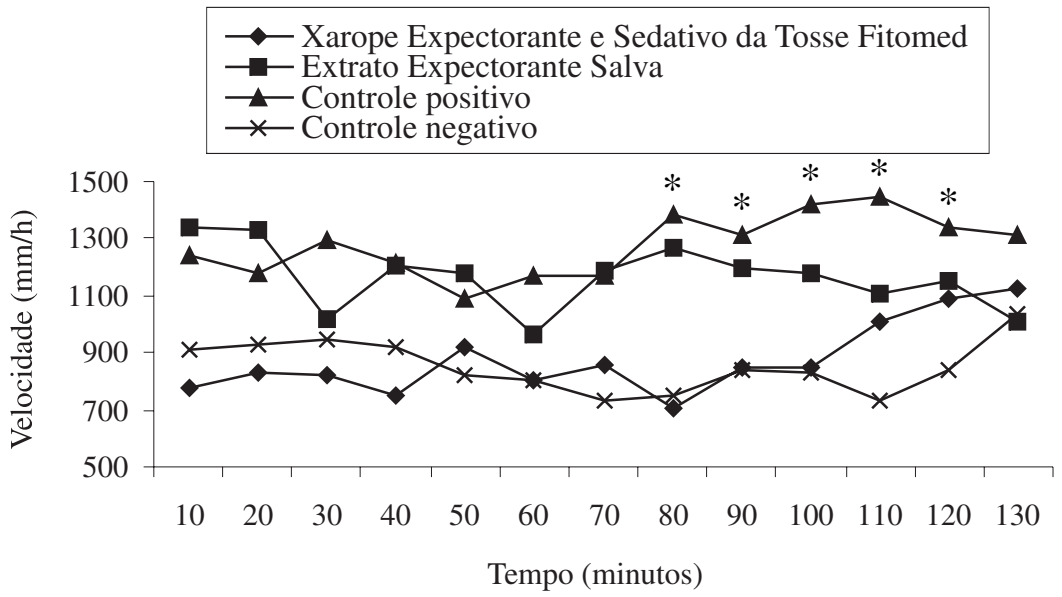

Figura 2. Velocidade do transporte mucociliar $(\mathrm{mm} / \mathrm{h})$ aferida através da adição de uma partícula marcadora (carvão ativado) na traquéia das codornas pertencentes ao pertencentes ao grupo com Extrato Expectorante Salva, Xarope Expectorante e Sedativo da Tosse Fitomed, grupo controle negativo tratado com o veículo (solução fisiológica) e grupo controle positivo tratado com erdosteína (Flusten). Os valores representam a média dos grupos em cada tempo medido. *Diferença estatisticamente significativa em relação ao controle negativo $(p<0,05)$. 
Os dois fitoterápicos estudados nesse trabalho foram avaliados frente a sua capacidade de modular a quantidade de secreção das vias aéreas, suprimir eventos de tosse e regular a velocidade de transporte mucociliar. Para tanto, as duas apresentações fitoterápicas foram avaliadas em cada modelo experimental proposto.

Quando avaliados perante ao modelo da avaliação da secreção das vias aéreas em ratos, nenhum deles apresentou a capacidade de modular a quantidade de secreção não diferindo do controle negativo.

Embora se tenha observado um aumento da concentração do corante no lavado broncoalveolar de ratos tratados com Extrato Expectorante Salva ${ }^{\circledR}$, os resultados não permitem concluir pela eficácia desse fitoterápico.

A propriedade de modular as secreções das vias aéreas é uma forma de facilitar a eliminação destas e auxiliar o organismo nos seus mecanismos defensivos da árvore respiratória [11]. Porém existem mecanismos diversos para que as ações defensivas sejam estimuladas e facilitadas.

A secreção do trato respiratório tem um papel importante na expectoração, porque as secreções estão intimamente relacionadas com a velocidade de transporte mucociliar [9].

Há autores que consideram que substâncias que aumentam a quantidade de secreções podem ser chamadas de substâncias com atividade secretagoga [5].

Quando uma substância apresenta propriedade de aumentar de forma consistente a secreção da árvore respiratória, este fato pode representar uma vantagem na terapêutica clínica [5].

$\mathrm{Na}$ avaliação dos fitoterápicos perante ao modelo de avaliação do reflexo da tosse induzido pelo ácido cítrico em cobaios, diferente do ocorrido no modelo anterior, eles apresentaram propriedades antitussígenas determinadas nesse modelo.

O porcentual de redução nos eventos de tosse apresentados pelos animais de cada grupo, permite fazer avaliações com relação a potência individual de cada fitoterápico.

O mecanismo de ação que justifique o efeito antitussígeno pode ser atribuído a cada constituinte presente nas plantas ou a associação de constituintes, e que serão discutidos a seguir.

De forma geral, os fitoterápicos atuam nos receptores periféricos da tosse e não através de mecanismos centrais conforme o ocorrido no grupo controle positivo [4]. Dessa forma, atribuímos que a inibição da tosse apresentada nos grupos de tratamento, ocorreu através da ação sobre as vias periféricas [3].
Essa ação ocorre em virtude da redução na transmissão dos impulsos originários principalmente dos RAR e dos receptores fibras C [19,24].

Quando avaliamos os fitoterápicos frente ao modelo de determinação da velocidade de transporte mucociliar em codornas, observamos que o nenhum dos dois fitoterápicos testados, apresentou eficácia no modelo, quando comparado com o grupo controle negativo, apesar do Extrato Expectorante Salva ${ }^{1}$, ter apresentado aumento da sua velocidade durante as medidas realizadas aos 70, 80, 90, 100, 110 e 120 minutos.

Substâncias que aumentam a velocidade do transporte mucociliar tem grande aplicabilidade terapêutica $[11,15]$.

Todas as plantas que compõem os dois fitoterápicos estudados apresentam indicações terapêuticas para o tratamento das patologias do aparelho respiratório [17].

As apresentações farmacêuticas fitoterápicas empregadas apresentaram ação sobre o aparelho respiratório, podendo ser denominadas de antitussígenos periféricos que atuam sobre os receptores da tosse localizados no epitélio das vias respiratórias [11].

$\mathrm{A}$ adição dos hidratantes às plantas utilizadas, provavelmente confere às formulações fitoterápicas as propriedades antitussígenas periféricas, além da ação resultante dos constituintes químicos das mesmas individualmente ou em associação.

Cada uma das plantas possui uma grande variedade de constituintes químicos como: óleos essenciais, taninos, terpenóides, flavonóides, saponinas e outros. A associação desses constituintes ou alguns especificamente possuem propriedades antiinflamatórias que provavelmente é a responsável por parte das ações desses fitoterápicos. Essa ação é expressa de forma consistente no modelo de avaliação do reflexo da tosse induzido pelo ácido cítrico.

É possível que a retirada de uma, duas, três ou mais plantas das formulações testadas, acarrete redução na eficácia do preparado. Portanto a exclusão de alguma delas nas associações, pode acarretar não só redução da eficácia, como a necessidade de aumentar a concentração das plantas remanescentes no preparado e com isso alterar os resultados relacionados a toxicidade do produto.

\section{CONCLUSÃO}

Os dois fitoterápicos utilizados nesse trabalho apresentaram efeito antitussígeno comprovado no mo- 
delo experimental de avaliação do reflexo da tosse induzido pelo ácido cítrico, sob as condições descritas, sugerindo eficácia para aplicabilidade clínica terapêutica, para esse fim.

Agradecimentos. Esse trabalho teve suporte financeiro da CAPES, CNPq, PROPESQ/UFRGS e FAPERGS.
NOTAS INFORMATIVAS

${ }^{1}$ Laboratório Industrial Vida e Saúde Ltda., Chapecó, SC, Brasil. ${ }^{2}$ Herbarium Laboratório Botânico Ltda., Colombo, PR, Brasil. ${ }^{3}$ Granja Asa Branca, Porto Alegre, RS, Brasil.

${ }^{4}$ Laboratório Eurofarma Ltda., São Paulo, SP, Brasil.

${ }^{5}$ Laboratório Enila, São Paulo, SP, Brasil.

${ }^{6}$ Virbac S/A, São Paulo, SP, Brasil.

${ }^{7}$ Nuclear, São Paulo, SP, Brasil.

\section{REFERÊNCIAS}

1 Banner A.S. 1986. Cough: physiology, evaluation and treatment. Lung. 164: 79-92.

2 Callegari-Jacques S.M. 2004. Bioestatística princípios e aplicações. São Paulo: Artmed Editora S.A., 255p.

3 Carr M.J. \& Ellis J.L. 2002. The study of airway primary afferent neuron excitability. Current Opinions in Pharmacology. 2: 216-219.

4 Chung K.F. \& Chang A.B. 2002. Therapy for cough: active agents. Pulmonary Pharmacology \& Therapeutics. 15: 335-338.

5 Disse B.G. \& Ziegler H.W. 1987. Pharmacodynamic mechanism and therapeutic activity of ambroxol in animal experiments. Respiration. 51: 15-22.

6 El-Hashim A.Z., Wyss D. \& Lewis C. 2004. Effect of a novel NK1 receptor selective antagonist (NKP608) on citric acid induced cough and airway obstruction. Pulmonary Pharmacology \& Therapeutics. 17: 11-18.

7 Fontana G.A., Lavorini F. \& Pistolesi M. 2002. Water aerosols and cough. Pulmonary Pharmacology \& Therapeutics. 15: 205-211.

8 Górniak S.L. 2002. Medicamentos com ação no sistema respiratório. In: Spinosa H.S., Górniak S.L. \& Bernardi M.M. (Eds). Farmacologia aplicada à medicina veterinária. 3.ed. Rio de Janeiro: Guanabara Koogan, pp.158-166.

9 Hosoe H., Kaise T., Isohama T.H., Kai H., Takahama K. \& Miyata T. 1999. Mucolytic and antitussive effects of erdosteine. Journal Pharmaceuthical Pharmacology. 51: 959-966.

10 Kohrogi H., Graf P.D., Sekizawa K. \& Borson B.D. 1989. Recombinant human enkephalinase (neutral endopeptidase) prevents cough induced by tachykinins in awake guinea pigs. Journal of Clinical Investigation. 82: 2063-2068.

11 Korolkovas A. 1999. Dicionário Terapêutico Guanabara. São Paulo: Guanabara Koogan SA, 721p.

12 Lapponi J.C. 2000. Estatística usando o Excel. 2.ed. São Paulo: Lapponi Treinamento e Editora, 451p.

13 Marchioni C.F, Moretti M., Muratori M., Casadei M.C., Guerzon M., Scuri R. \& Fregnan G.B. 1990. Effects of erdosteine on sputum biochemical and rheological properties: pharmacokinetics in chronic obstructive lung disease. Lung. 168: 285-293.

14 Marriott C., Readman A.S. \& Barrett-Bee K. 1983. The effect of some drugs on the biophysical and biochemical properties of pig tracheal mucus. European Journal Respiratory Disease. 64: 441-443.

15 Miyata T., Kai H., Isohama Y. \& Takahama K. 1998. Current opinion of muco active drug research: strategies and problems. European Respiration Journal. 11: 480-491.

16 O'Connell F. 2002. Central pathways for cough in man - unanswered questions. Pulmonary Pharmacology \& Therapeutics. 15: 295-301.

17 Oliveira F., Saito M.L. \& Garcia L.O. 1993. Caracterização cromatográfica em camada delgada do extrato fluido de guaco - Mikania glomerata Sprengel. LECTA -USF. 11: 43-56 .

18 Pantaleo T., Bongianni F. \& Mutolo D. 2002. Central nervous mechanisms of cough. Pulmonary Pharmacology \& Therapeutics. 15: 227-233.

19 Ricciardolo F.L.M. 2001. Mechanisms of citric acid-induced bronchoconstriction. The American Journal of Medicine. 111: $18 \mathrm{~s}-24 \mathrm{~s}$.

20 Wanner A. 1986. Effect of ibatropium bromide on airway mucociliary function. The American Journal of Medicine. 81: 23-27.

21 Wanner A. 1977. Clinical aspects of mucociliary transport. American Review of Respiratory Disease. 116: 73-125.

22 Widdicombe J. 2002. Neuroregulation of cough: implications for drug therapy. Current Opinion in Pharmacology. 2: 256-263.

23 Zar J.H. 1999. Biostatistical analysis. 4th edn. New Jersey: Prentice Hall, 123p.

24 Ziment I. \& O'Connell F. 2002. Summary: clinical needs for cough therapy. Pulmonary Pharmacology \& Therapeutics. 15: 293-294.

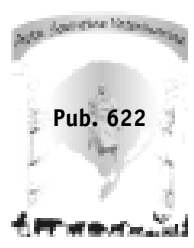

